\title{
PONTRYAGIN DUALITY IN $K$-THEORY
}

\author{
J. W. VICK
}

Let $K^{*}$ and $K_{*}$ denote the $Z_{2}$-graded cohomology and homology theories arising from the unitary spectrum. For $G$ a finite group denote by $B G$ a classifying space for $G$. In [13] it was observed as a direct result of calculation of the groups that for $G=Z_{p}, p$ a prime, $\tilde{K}^{\circ}\left(B Z_{p}\right)$ is the Pontryagin dual of $K_{1}\left(B Z_{p}\right)$. That is, $\tilde{K}^{\circ}\left(B Z_{p}\right)$ is a compact group isomorphic to the character group of the discrete group $K_{1}\left(B Z_{p}\right)$. Our purpose is to prove the following:

Theorem. For any finite group $G$, the compact group $\tilde{K}^{\circ}(B G)$ is isomorphic to the character group of the discrete group $K_{1}(B G)$.

In view of Atiyah's computation [3] of $\tilde{K}^{\circ}(B G)$ this determines $K_{1}(B G)$. To complete the picture it is observed that $\tilde{K}_{\circ}(B G)=0$ corresponding to the determination in [3] that $K^{1}(B G)=0$. However, the theorem does not generalize to compact Lie groups; in particular it fails when $G=S^{1}$.

The preliminaries necessary to the proof are, for the most part, well known facts. Thus they are presented in as concise a manner as possible. Generalities are avoided unless essential to the proof.

1. Limits. Suppose

$$
G_{1} \stackrel{\phi_{1}}{\leftarrow} G_{2} \stackrel{\phi_{2}}{\leftarrow} G_{3} \stackrel{\phi_{3}}{\leftarrow} \ldots
$$

is an inverse system of abelian groups. Define a homomorphism

$$
\Phi: \prod_{i=1}^{\infty} G_{i} \rightarrow \prod_{i=1}^{\infty} G_{i}
$$

by $\Phi\left(g_{1}, g_{2}, g_{3}, \cdots\right)=\left(g_{1}-\phi_{1}\left(g_{2}\right), g_{2}-\phi_{2}\left(g_{3}\right), \cdots\right)$. Then proj lim $G_{i}$ $=\operatorname{ker} \Phi$ and $\lim ^{1}\left\{G_{i}\right\}=\operatorname{coker} \Phi$.

The standard tool relating the cohomology groups of a CW complex of finite type to the groups of its finite subcomplexes is the Milnor result [8].

(1.1) Let $X_{1} \subset X_{2} \subset \cdots \subset X$ be a filtration of $X$ by finite subcomplexes, and $h^{*}$ an additive cohomology theory. Then for any $p,\left\{\tilde{h}^{p}\left(X_{i}\right)\right\}$ gives an inverse system and the following is exact

$$
0 \rightarrow \lim ^{1}\left\{\tilde{h}^{p-1}\left(X_{i}\right)\right\} \rightarrow \tilde{h}^{p}(X) \rightarrow \operatorname{proj} \lim \left\{\tilde{h}^{p}\left(X_{i}\right)\right\} \rightarrow 0 .
$$

Received by the editors June 16, 1969. 
Following [4], [3] we say that an inverse system $\left\{G_{i}, \phi_{i}\right\}$ satisfies the Mittag-Leffler condition (M-L) if for each $n$ there exists an integer $N$ such than when $k \geqq N$, the image of $\phi_{k, n}=\phi_{n} \circ \cdots \circ \phi_{k-1}$ equals the image of $\phi_{N, n}=\phi_{n} \circ \cdots \circ \phi_{N-1}$. A stronger condition, denoted by Condition (F) in [3], is that for each $n$ there exists an integer $N$ with the image of $\phi_{N, n}$ finite.

This condition is related to the preservation of exactness in inverse limits in the following way [4], [3]:

(1.2) Let $0 \rightarrow\left\{A_{i}\right\} \rightarrow\left\{B_{i}\right\} \rightarrow\left\{C_{i}\right\} \rightarrow 0$ be an exact sequence of inverse systems. If $\left\{A_{i}\right\}$ satisfies (M-L) then

$$
0 \rightarrow \operatorname{proj} \lim \left\{A_{i}\right\} \rightarrow \operatorname{proj} \lim \left\{B_{i}\right\} \rightarrow \operatorname{proj} \lim \left\{C_{i}\right\} \rightarrow 0
$$

is exact.

Finally there is the relationship between this condition and $\lim ^{1}$ as observed in [11], [1]

(1.3) If $\left\{G_{i}, \phi_{i}\right\}$ satisfies (M-L) then $\lim ^{1}\left\{G_{i}\right\}=0$.

2. $K$-theory. The generalized homology and cohomology theories we employ are those arising from the unitary spectrum $U$ (see [6] for details). As observed in [6] there is a pairing of the unitary spectrum with itself $(U, U) \rightarrow U$ in the sense of Whitehead [15]. This leads to various products on the theories. In particular there is the Kronecker index [6]

$$
\tilde{K}^{p}(X) \otimes \tilde{K}_{p}(X) \rightarrow \tilde{K}^{\circ}\left(S^{\circ}\right) \approx Z
$$

which gives natural homomorphisms

$$
\tilde{K}^{p}(X) \rightarrow \operatorname{Hom}\left(\tilde{K}_{p}(X), Z\right) \text { and } \tilde{K}_{p}(X) \rightarrow \operatorname{Hom}\left(\tilde{K}^{p}(X), Z\right) .
$$

These homomorphisms provide a useful tool in studying the duality between $K^{*}$ and $K_{*}$ as expressed by the following unpublished result of D. Anderson [2] (see also [14]):

(2.1) For any finite $C W$ complex $X$ there is a natural exact sequence

$$
0 \rightarrow \operatorname{Ext}\left(\tilde{K}_{n-1}(X), Z\right) \rightarrow \tilde{K}^{n}(X) \rightarrow \operatorname{Hom}\left(\tilde{K}_{n}(X), Z\right) \rightarrow 0 .
$$

There is also the corresponding sequence [14].

(2.2) For any finite $C W$ complex $X$ there is a natural exact sequence

$$
0 \rightarrow \operatorname{Ext}\left(\tilde{K}^{n+1}(X), Z\right) \rightarrow \widetilde{K}_{n}(X) \rightarrow \operatorname{Hom}\left(\tilde{K}^{n}(X), Z\right) \rightarrow 0 .
$$

Finally we observe as in [6] that the cohomology theory $K^{*}$ is additive in the sense of Milnor.

3. Classifying spaces. Let $G$ be a finite group. We use the Milnor construction [9] to give a classifying space $B G$. Recall that there is a 
filtration of the total space $E_{1} \subset E_{2} \subset \cdots \subset E$ by finite $C W$ complexes where $E_{n}$ is the $(n+1)$-fold join of copies of $G$. $G$ acts freely on $E$ in such a way that each $E_{n}$ is invariant. So in $B G=E / G$ there is the corresponding filtration by quotient spaces $B_{1} \subset B_{2} \subset \cdots \subset B G$.

Following the approach in [9] define a function

$$
f: C E_{n} \rightarrow B_{n+1} \text {, }
$$

where $C E_{n}$ is the cone over $E_{n}$, as follows: Consider $E_{n+1}$ as $G \circ E_{n}$ and write a point $x$ in the form $x=t g+(1-t) \bar{g}$ where $g \in G$ and $\bar{g} \in E_{n}$. Denote by $[x]$ the image of $x$ in $B_{n+1}$ under the quotient map. Then for $(t, \bar{g}) \in C E_{n}$ define

$$
f(t, g)=[t \cdot e+(1-t) \bar{g}]
$$

where $e$ is the identity of $G$. $f$ is well defined and continuous.

Identifying $E_{n}$ with the base in $C E_{n}$, one can observe that

$$
\left.f\right|_{E_{n}}: E_{n} \rightarrow B_{n} \subset B_{n+1}
$$

is the quotient map and

$$
f: C E_{n}-E_{n} \rightarrow B_{n+1}-B_{n}
$$

is a homeomorphism.

Thus for any $p$ there are isomorphisms

$$
\tilde{K}_{p}\left(E_{n}\right) \approx \tilde{K}_{p+1}\left(S E_{n}\right) \approx K_{p+1}\left(C E_{n}, E_{n}\right) \stackrel{f_{*}}{\underset{\approx}{\approx}} K_{p+1}\left(B_{n+1}, B_{n}\right)
$$

whose composition we denote by $\psi$.

Then consider the exact sequence of the pair $\left(B_{n+1}, B_{n}\right)$

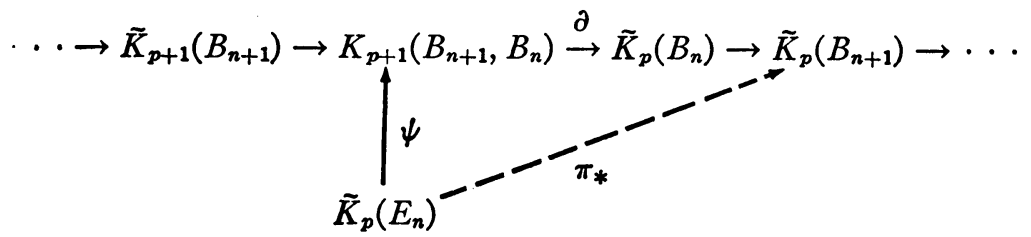

and observe that the triangle commutes where $\pi$ is the quotient map.

Let $Q$ be the rationals and consider

$$
\pi_{*} \otimes 1: \widetilde{H}_{p}\left(E_{n}\right) \otimes Q \rightarrow \widetilde{H}_{p}\left(B_{n}\right) \otimes Q
$$

where $H_{*}$ is singular homology. By results of Serre [12-appendix] this is an epimorphism. Using the spectral sequence of Dold [5] for the spaces $E_{n}$ and $B_{n}$ we can conclude that

$$
\pi_{*} \otimes 1: \tilde{K}_{p}\left(E_{n}\right) \otimes Q \rightarrow \tilde{K}_{p}\left(B_{n}\right) \otimes Q
$$


is an epimorphism. Thus we have

(3.1) The cokernel of $\pi_{*}: \tilde{K}_{p}\left(E_{n}\right) \rightarrow \widetilde{K}_{p}\left(B_{n}\right)$ is finite for each $p$ and $n$.

A similar approach for $K^{*}$ yields the following

(3.2) The kernel of $\pi^{*}: \widetilde{K}^{p}\left(B_{n}\right) \rightarrow \widetilde{K}^{p}\left(E_{n}\right)$ is finite for each $p$ and $n$.

As an immediate corollary we have the result of Atiyah [3]

(3.3) The image of $i^{*}: \widetilde{K}^{p}\left(B_{n+1}\right) \rightarrow \widetilde{K}^{p}\left(B_{n}\right)$ is finite for each $p$ and $n$, and in particular the inverse system $\left\{\widetilde{K}^{p}\left(B_{n}\right)\right\}$ satisfies Condition $(\mathrm{F})$.

Finally observe that $E_{n}$ has the homotopy type of a wedge of $n$ spheres, thus $K_{1}\left(E_{2 n}\right), \tilde{K}_{\circ}\left(E_{2 n+1}\right), K^{1}\left(E_{2 n}\right)$ and $\widetilde{K}^{\circ}\left(E_{2 n+1}\right)$ are all zero. This implies

(3.4) The groups $K_{1}\left(B_{2 n}\right), \tilde{K}_{\circ}\left(B_{2 n+1}\right), K^{1}\left(B_{2 n}\right)$ and $\tilde{K}^{\circ}\left(B_{1 n+1}\right)$ are all finite.

4. Proof of theorem. Recall that for a locally compact commutative group $H$, the dual or character group $\mathbf{X}(H)$ is the group of homomorphisms of $H$ into the real numbers modulo the integers. $\mathrm{X}(H)$ is then a locally compact commutative group under the compactopen topology.

There is the fundamental Pontryagin duality theorem [10]

(4.1) If $H$ is a locally compact commutative group, then the natural mapping $H \rightarrow \mathrm{X}(\mathrm{X}(H))$ is an isomorphism.

Let

$$
H_{1} \stackrel{\phi_{1}}{\rightarrow} H_{2} \stackrel{\phi_{2}}{\rightarrow} H_{3} \rightarrow \cdots
$$

be a direct system of discrete abelian groups. Then another wellknown fact [7, Chapter 2] is that

(4.2) $\mathrm{X}\left(\operatorname{inj} \lim \left\{H_{i}\right\}\right) \approx \operatorname{proj} \lim \left\{\mathrm{X}\left(H_{i}\right)\right\}$.

The connection between this and the previous sections is given by the following lemma

(4.3) For $H$ a finite abelian group there is a natural isomorphism

$$
\phi: \operatorname{Ext}(H, Z) \rightarrow \mathrm{X}(H) .
$$

To prove this consider the injective resolution of $Z$ given by

$$
0 \rightarrow Z \rightarrow R \rightarrow R / Z \rightarrow 0 .
$$

Then $\operatorname{Ext}(H, Z)$ is naturally isomorphic to the cokernel of

$$
\operatorname{Hom}(H, R) \rightarrow \operatorname{Hom}(H, R / Z) \text {. }
$$

But since $H$ is finite, $\operatorname{Hom}(H, R)=0$ and (4.3) follows.

Now denote by $K_{p}(X)$ the torsion subgroup of $K_{p}(X)$. Recall that by (3.4) $K_{1}\left(B_{2 n}\right)=K_{1}\left(B_{2 n}\right)$. From the Milnor sequence (1.1) and the results (3.3) and (1.3) we know that 


$$
\tilde{K}^{\circ}(B G) \approx \operatorname{proj} \lim \tilde{K}^{\circ}\left(B_{n}\right) .
$$

The Anderson sequence (2.1) for the spaces $\left\{B_{n}\right\}$ gives an exact sequence of inverse systems. By the application of (3.3) and (1.2) we have the exactness of

$0 \rightarrow$ proj $\lim \operatorname{Ext}\left(K_{1}\left(B_{n}\right), Z\right) \rightarrow \operatorname{proj} \lim \tilde{K}^{\circ}\left(B_{n}\right)$

$$
\rightarrow \operatorname{proj} \lim \operatorname{Hom}\left(\tilde{K}_{\circ}\left(B_{n}\right), Z\right) \rightarrow 0 .
$$

By (3.4) proj $\lim \left\{\operatorname{Hom}\left(\tilde{K}_{\circ}\left(B_{n}\right), Z\right)\right\}=0$ so that

$$
\operatorname{proj} \lim \left\{\tilde{K}^{\circ}\left(B_{n}\right)\right\} \approx \operatorname{proj} \lim \left\{\operatorname{Ext}\left(K_{1}\left(B_{n}\right), Z\right)\right\}
$$

and obviously

$$
\operatorname{proj} \lim \left\{\operatorname{Ext}\left(K_{1}\left(B_{n}\right), Z\right)\right\} \approx \operatorname{proj} \lim \left\{\operatorname{Ext}\left(K_{1}\left(B_{n}\right), Z\right)\right\} .
$$

By the naturality of the isomorphism in (4.3)

$$
\operatorname{proj} \lim \left\{\operatorname{Ext}\left(K_{1}\left(B_{n}\right), Z\right)\right\} \approx \operatorname{proj} \lim \left\{\mathrm{X}\left(K_{1}\left(B_{n}\right)\right)\right\},
$$

and by (4.2)

$$
\operatorname{proj} \lim \left\{\mathrm{X}\left(K_{1}\left(B_{n}\right)\right)\right\} \approx \mathrm{X}\left(\operatorname{inj} \lim \left\{K_{1}\left(B_{n}\right)\right\}\right) .
$$

Finally, since the sequence $\left\{K_{1}\left(B_{2 n}\right)\right\}$ is cofinal,

$$
\mathrm{X}\left(\operatorname{inj} \lim \left\{K_{1}\left(B_{n}\right)\right\}\right) \approx \mathrm{X}\left(\operatorname{inj} \lim \left\{K_{1}\left(B_{n}\right)\right\}\right) \approx \mathrm{X}\left(K_{1}(B G)\right) .
$$

This completes the proof that $\tilde{K}^{\circ}(B G) \approx \mathrm{X}\left(K_{1}(B G)\right)$.

To determine $\tilde{K}_{\circ}(B G)$ we consider the exact sequence of direct systems given by applying (2.2) to the spaces $B_{n}$. This yields an isomorphism

$$
\operatorname{inj} \lim \left\{\operatorname{Ext}\left(K^{1}\left(B_{n}\right), Z\right)\right\} \approx \operatorname{inj} \lim \left\{\tilde{K}_{\circ}\left(B_{n}\right)\right\} \approx \tilde{K}_{\circ}(B G) .
$$

Now it has been shown by Atiyah [3] that $K^{1}(B G)=0$; and since $K^{1}(B G) \approx \operatorname{proj} \lim \left\{K^{1}\left(B_{n}\right)\right\}$, it is not hard to see that inj $\lim \left\{\operatorname{Ext}\left(K^{1}\left(B_{n}\right), Z\right)\right\}=0$. Thus $\tilde{K}_{\circ}(B G)=0$.

In view of the determination in [3] of $\tilde{K}^{\circ}(B G)$ as a completion of the representation ring $R(G)$, this duality determines $K_{*}(B G)$. In some cases this dual group has a particularly nice form (see [13]) which readily lends itself to applications. It would be of interest to know if this duality results from some natural pairing of the theories into the rational numbers modulo the integers. This seems to require a more geometric interpretation of $K_{*}(X)$. Another point of interest is whether this duality arises in some natural way from the analytical definition of $K$-theory using Fredholm operators. 


\section{REFERENCES}

1. D. W. Anderson and L. Hodgkin, The K-theory of Eilenberg-MacLane complexes, Topology 7 (1968), 317-329. MR 37 \#6924.

2. D. W. Anderson, Universal coefficient theorems for $K$-theory (unpublished).

3. M. F. Atiyah, Characters and cohomology of finite groups, Inst. Hautes Etudes Sci. Publ. Math. No. 9 (1961), 23-64. MR $26 \# 6228$.

4. J. Dieudonné and A. Grothendieck, Éléments de géométrie algébrique. III : Études cohomologique des faisceaux cohérents. I, Inst. Hautes Êtudes Sci. Publ. Math. No. 11 (1961), MR 36 \#177c.

5. A. Dold, Relations between ordinary and extraordinary homology, Colloq. Algebraic Topology, Matematisk Institute, Aarhus Universitet, Aarhus, 1962. MR 26 \#3565.

6. A. Hattori, Integral characteristic numbers for weakly almost complex manifolds, Topology 5 (1966), 259-280. MR 33 \#742.

7. S. Lefschetz, Algebraic topology, Amer. Math. Soc. Colloq. Publ., vol. 27, Amer. Math. Soc., Providence, R. I., 1942. MR 4, 84.

8. J. Milnor, On axiomatic homology theory, Pacific J. Math. 12 (1962), 337-341. MR 28 \#2544.

9. - Construction of universal bundles. II, Ann. of Math. (2) 63 (1956), 430436. MR 17, 1120.

10. L. Pontrjagin, Topological groups, Princeton Univ. Press, Princeton, N. J., 1939. MR 1, 4.4.

11. J. E. Roos, Sur les foncteurs dérivés de lim. Applications, C.R. Acad. Sci. Paris 252 (1961), 3702-3704. MR 24 \#A1938.

12. J. P. Serre, Homologie singulière des espaces fibrés. Applications, Ann. of Math. (2) 54 (1951), 425-505. MR 13, 574.

13. J. W. Vick, An application of $K$-theory to equivariant maps, Bull. Amer. Math. Soc. 75 (1969), 1017-1019.

14. - Thesis, University of Virginia, Charlottesville, Va., 1968.

15. G. W. Whitehead, Generalized homology theories, Trans. Amer. Math. Soc. 102 (1962), 227-283. MR $25 \# 573$.

\section{Princeton University}

